\title{
Adaptive mobile firefighting resources: stochastic dynamic optimization of international cooperation
}

\begin{abstract}
Forest fires cause severe problems in many countries. Forest fire areas in nine European countries are investigated with respect to yearly averages, standard deviations and correlations between nations. In the region IFPS (Italy, France, Portugal and Spain), the average yearly burned area during the years 2010 to 2018 was 313.4 kha and in FGLNS (Finland, Germany, Latvia, Norway and Sweden) the corresponding area was only $7.6 \mathrm{kha}$. The correlations between the regions are strictly negative and the correlations within the regions are strictly positive.

Since forest fires usually do not occur in every country at the same time, there is a potential expected gain from international cooperation, where easily mobile firefighting resources such as water bombing airplanes are moved between nations. A general stochastic dynamic programming approach to adaptive moves of such resources is defined and suggested. General properties of the solution are derived. A particular version of the model is created and analytical derivations are performed. It is demonstrated that the expected objective function value, the expected present value of total costs, is a strictly increasing function of the fire correlation between nations. Adaptive moves of mobile resources between the regions IFPS and FGLNS have the advantage of negative correlations between these regions. Some adaptive moves can also be motivated within the regions even with positive correlations, thanks to the low costs of short moves.
\end{abstract}

Volume 6 Issue 4 - 2020

Peter Lohmander

Optimal Solutions in Cooperation with Linnaeus University, Sweden

Correspondence: Peter Lohmander, Optimal Solutions in Cooperation with Linnaeus University, Hoppets Grand 6, SE 903 34 Umea, Sweden, Email peter@lohmander.com

Received: October 17, 2020 | Published: November 17, 2020

\section{Introduction}

Forest fires cause severe problems, locally and globally. A general reference on present forest conditions is FAO (2020). ${ }^{1}$ Abatzoglou and Williams, et al $2016,{ }^{2}$ Coogan, et al $2019^{3}$ and Williams, et al $2019,{ }^{4}$ describe and give warnings for increasing wildfire frequencies as a result of global warming. The fire emissions of $\mathrm{CO}_{2}$, have international consequences via the effect on global warming. The released $\mathrm{CO}_{2}$ moves without friction across the globe. Recent studies and results concerning $\mathrm{CO}_{2}$ dynamics and rational forest management are found in Lohmander (2020a, 2020b and 2020c) ${ }^{5-7}$ Countries close to the equator mostly have much larger and more dramatic forest fires than countries closer to the poles. Table 1 , shows this very clearly, where it is found that the European countries in the south: Italy, France, Portugal and Spain, denoted "IFPS", on average, together, have the average yearly burned area of 313400 hectares. The corresponding figure for the five European countries in the north, Finland, Germany, Latvia, Norway and Sweden, "FGLNS", is only 7600 hectares. Hence, the average burned forest area in the southern countries is more than 41 times larger than in the north. Of course, the forest areas are not identical and many other things differ between the regions. Still, it is quite clear that the forest fire problems are much worse in warmer climates. Forest fires normally occur during the warm season and are reduced during the winter. When there is winter north of the equator, there is summer further south. Hence, fires are more common south of the equator during the period when they are less common in the north and vice versa. This periodic pattern can be used to move firefighting capacity over the equator in order to utilize it more rationally. Cave, $2019^{9}$ discusses the firefighting resource problem including Australia, California and many other regions of the world. Global warming has made the problems worse. The firefighting aircrafts are now more often included in international contracts where these move between nations. The Maritime Executive, $2020^{10}$ presents a suggestion where
Canada and Australia share 14 water bombers and use a special ship to move the planes between the nations in order to handle the fire seasons on both sides of the equator. Firefighting resources can also be shared between countries on the same side of the equator. Fire \& Rescue World News, 2018 ${ }^{11}$ describes how Sweden during 2018 requested help from the European Union. The Swedish fires were much larger than earlier years and the Swedish firefighting resources were insufficient. Italy was able to assist with water bombers. Later, Mason, $2020^{12}$ report that Sweden expands the water bomber capacity, intended for operations in Sweden and other parts of Northern Europe. April to September is considered to be the relevant fire season there. This paper focuses on optimization of firefighting with stochastic fires and adaptive moves of firefighting units, in particular water bombing aircrafts, between nations. First, statistics from nine European countries will be used to investigate the stochastic properties of fires, including average burned areas, standard deviations and correlations across countries and regions. Then, a general stochastic dynamic programming model for optimal adaptive allocation of firefighting units across nations, will be developed. General conclusions will be derived based on the mathematical model, including the effects on the expected total result of the number of countries in the cooperation contract and the effects of fire correlations between regions.

In Table 2, we find the correlations between the burned areas in the different countries. The correlations between the countries in the southern region, IFPS, are all strictly positive. This part of the correlation table has been marked by yellow. The correlations between the countries in the northern region, FGLNS, are all strictly positive. This part of the correlation table has been marked by red. The turquoise section of Table 2 shows that all correlations of burned areas of countries in IFPS with countries in FGLNS, are strictly negative. This pattern is repeated if we look at the correlation table only containing the two groups IFPS and FGLNS, which is found in Table 3. 
Table I The average yearly burned areas in different countries and regions during nine years (from 20I0 until 20I8). The standard deviations and the relative standard deviations have also been calculated."IFPS" denotes the region including Italy, France, Portugal and Spain. "FGLNS" denotes the region including Finland, Germany, Latvia, Norway and Sweden. Source of the list of burned areas: San-Miguel-Ayanz et al., $2019^{8}$

\begin{tabular}{llll}
\hline & Average Burned Area (kha) & Standard Deviation (kha) & Relative Standard Deviation \\
\hline Italy & 62,3 & 43,1 & 0,7 \\
France & 10,9 & 6,8 & 0,6 \\
Portugal & 144,6 & 156,3 & 1,1 \\
Spain & 95,7 & 66,3 & 0,7 \\
Finland & 0,5 & 0,4 & 0,7 \\
Germany & 0,5 & 0,7 & 1,3 \\
Latvia & 0,6 & 0,9 & 1,5 \\
Norway & 0,8 & 1,1 & 1,3 \\
Sweden & 5,1 & 8,5 & 1,7 \\
IFPS & 313,4 & 240,1 & 0,8 \\
FGLNS & 7,6 & $\mathrm{II}, \mathrm{I}$ & 1,5
\end{tabular}

Table 2 Correlations of burned areas in different countries during nine years (from 2010 until 20I8). The original data that were used to calculate these correlations are available in the official statistics by San-Miguel-Ayanz et al., 20198

\begin{tabular}{|c|c|c|c|c|c|c|c|c|c|}
\hline & Italy & France & Portugal & Spain & Finland & Germany & Latvia & Norway & Sweden \\
\hline Italy & 1,000 & & & & & & & & \\
\hline France & 0,634 & 1,000 & & & & & & & \\
\hline Portugal & 0,657 & 0,859 & 1,000 & & & & & & \\
\hline Spain & 0,944 & 0,464 & 0,482 & 1,000 & & & & & \\
\hline Finland & $-0,492$ & $-0,313$ & $-0,230$ & $-0,65 I$ & 1,000 & & & & \\
\hline Germany & $-0,349$ & $-0,238$ & $-0,184$ & $-0,369$ & 0,666 & 1,000 & & & \\
\hline Latvia & $-0,459$ & $-0,291$ & $-0,280$ & $-0,467$ & 0,742 & 0,951 & 1,000 & & \\
\hline Norway & $-0,427$ & $-0,081$ & $-0,158$ & $-0,531$ & 0,682 & 0,824 & 0,864 & 1,000 & \\
\hline Sweden & $-0,464$ & $-0,377$ & $-0,356$ & $-0,521$ & 0,894 & 0,767 & 0,888 & 0,762 & 1,000 \\
\hline
\end{tabular}

Table 3 Correlations of burned areas in two regions during nine years (from 2010 until 2018). Source of empirical data observations: San-Miguel-Ayanz et al., $2019^{8}$

IFPS

FGLNS

IFPS

FGLNS

\begin{tabular}{|l|l|}
\hline I \\
\hline$-0,458$ & \\
\hline
\end{tabular}

Figure 1 shows that the strictly negative correlation between the burned areas in IFPS and FGLNS can be understood from the empirical observations from year 2010 until 2018. During this period, we note that the fire areas in the north have been comparatively large when they have been comparatively small in the south and vice versa. Still, all of the countries are located north of the equator. We also observe that the burned areas differ very much between years. Hence, it is very likely that we can obtain better use of the firefighting resources in Europe if these can be easily moved between the regions IFPS and FGLNS. The fire areas and the absolute variations are much larger in the south than in the north. So, even if the firefighting capacity would not be optimized for Europe, but for the regions in isolation, we can expect that IFPS many years would have very large excess capacity. Some parts of this excess capacity could be sent to FGLNS if needed without causing problems in IFPS.

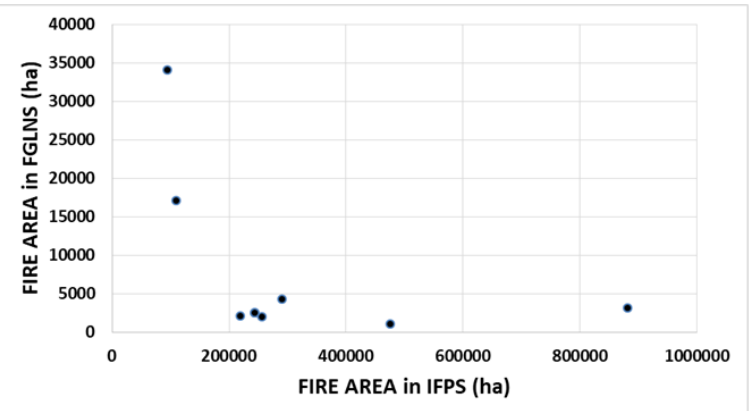

Figure I Observations of combinations of burned areas in two regions during nine years (from 2010 until 2018). The raw fire data are available in San-Miguel-Ayanz et al., 2019.

\section{Materials and methods}

The optimization problem is defined as a stochastic dynamic programming problem. Hence, it is possible to sequentially optimize the decisions, based on the latest information about the stochastic events and the state of the complete system. This methodology is absolutely necessary in this problem in order to make it relevant, since the fires are truly stochastic in nature and the central problem is to act in a way that is optimal and adaptive. In equation (1), we find the 
decision problem at the time horizon. It may seem to be unrealistic to have a time horizon, but since this can be defined at a point in time many years from the present, it does not influence the decisions and values in earlier periods more than we want it to. The time period is $t$, the time horizon is $T$ and $r$ is the rate of interest in the capital market. $f_{k, t}$ is the state in country (or region) $k$ at time $t .1 \leq k \leq n$. $1 \leq f_{k, t} \leq F_{k, t}, \forall k, t$. Note that the state in the optimization problem is multidimensional and includes all relevant information, such as the fire states in all different countries, available local firefighting resources, infrastructure and so on. $i_{t}$ is the entering state (the location) of a mobile firefighting resource unit. In the analysis in this paper, we consider $i_{t}$ to have dimension 1 . The problem may be generalized to multidimensional versions if necessary. The adaptive decision is to move this resource to location $j_{t}$. In this paper, we consider $j_{t}$ with dimension 1. This can be generalized. The feasible set is $J_{T}$ , which is a function of the entering state. $C$ denotes the sum of all costs in a particular period. This includes costs of moving firefighting equipment, costs of forests destroyed by fires and other associated costs. $\phi$ is the expected present value of all costs, in the present and future periods, as a function the time period and the entering state, which may be described in detail as the combination of the initial location(s) of mobile resources and the states in all countries, $f_{1, t}, f_{n, t}$.

\section{Definitions:}

At the time horizon, $t=T$, we have:

$$
\begin{gathered}
\phi\left(T, i_{T}, f_{1, T}, ., f_{n, T}\right)= \\
=\min _{j T \in J_{T}\left(i_{T}\right)}\left\{e^{-r T} C\left(T, i_{T}, j_{T}, f_{1, T}, ., f_{n, T}\right)\right\} \\
\forall i_{T}, f_{1, T}, ., f_{n, T}
\end{gathered}
$$

The complete problem is solved via Bellman backward recursion. When we go from one period to the next, it is necessary to calculate probabilities to move between states. $\tau($.) denotes the conditional transition probabilities.

$$
\begin{aligned}
& \text { For }\left.t\right|_{0 \leq t<T} \text {, we have: } \\
& \phi\left(t, i_{t}, f_{1, t}, ., f_{n, t}\right)=
\end{aligned}
$$

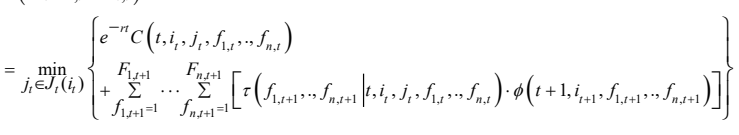

$$
\begin{aligned}
& \left.\forall t\right|_{0 \leq t<T}, i_{t}, f_{1, t},, f_{n, t}
\end{aligned}
$$

The entering location in the following period is determined by the move in this period.

$$
i_{t+1}=j_{t}
$$

The states in the different countries $k$ in the following period, are functions of time, the earlier states and moves, and stochastic events, $\varepsilon_{k, t+1}$.

$$
f_{k, t+1}=f_{k, t+1}\left(t+1, i_{t}, j_{t}, f_{k, t}, \varepsilon_{k, t+1}\right)
$$

The level of detail described in equations (1) to (4) is typically necessary in numerical versions of this optimization problem. An introduction to stochastic dynamic programming is given in Winston WL, et al., 2004. ${ }^{13}$ Alternative solution algorithms in cases of large dimensionality and alternative formulations are described in Lohmander, et al.,2017 ${ }^{14}$ and Lohmander, et al.,2018. ${ }^{15}$ Now, however, some general properties and results will be investigated and determined. In order to make the exposition easy to follow, the notation is simplified in the following steps:

$$
\begin{gathered}
j=j_{t} J=J_{t}\left(i_{t}\right) \\
\psi\left(j_{t}\right)=e^{-r t} C\left(t, i_{t}, j_{t}, f_{1, t}, ., f_{n, t}\right) \\
\beta\left(j_{t}\right)=\sum_{f_{1, t+1}=1}^{F_{1, t+1}} \ldots \sum_{f_{n, t+1}=1}^{\sum_{n+1}}\left[\tau\left(f_{1, t+1}, ., f_{n, t+1} \mid t, i_{t}, j_{t}, f_{1, t}, ., f_{n, t}\right) \cdot \phi\left(t+1, i_{t+1}, f_{1, t+1}, ., f_{n, t+1}\right)\right]
\end{gathered}
$$

Hence, we want to minimize according to expression (8):

$$
\phi=\min _{j \in J}\{\psi(j)+\beta(j)\}
$$

The problem may be even more simplified with the following objective function:

$$
\begin{aligned}
\varphi(j) & =\psi(j)+\beta(j) \\
\phi & =\min _{j \in J}\{\varphi(j)\}
\end{aligned}
$$

In order to determine the minimum, we may use the linear programming approach:

$$
\begin{aligned}
& \phi=\max \chi \\
& \text { s.t. } \quad \chi \leq \varphi(j) \forall j
\end{aligned}
$$

Notation is once again simplified:

$$
\begin{aligned}
& \alpha_{j}=\varphi(j), 1 \leq j \leq K \\
& \phi=\max \chi
\end{aligned}
$$$$
\text { s.t. }
$$

$$
\chi \leq \alpha_{j}, 1 \leq j \leq K
$$

From Figure 2 it is clear that the objective function nonstrictly decreases if the number of possible moves of the firefighting resource increases. This is stated in equation (14). This is one strong motive why we should consider alternative options to expand international cooperation in the firefighting operations. (Note that possibly increasing costs of administration are ignored here.)

$$
(\Delta K>0) \Rightarrow(\Delta \phi \leq 0)
$$

If the fire problems in two countries have strong positive correlation, the fire areas and the economic importance of using constrained firefighting resources, are usually high in both countries simultaneously. Hence, in times when fire conditions are difficult, the marginal values of the resource are high in both countries. In other periods with limited fire problems, the marginal values of the resource are low in both countries. Figure 3 illustrates the situation with strongly positive correlation. In case (a), the economic effect of sending the firefighting resource to region 1 gives a very low objective function value. The alternative destination, region 2, also gives a low objective function value. Region 1 is however the best alternative. We may assume that the difference between these two options is caused by the fact that the resources initially are located to region 1 and that a transport of the resources from region 1 to region 2 implies that we get increased costs. Region 1 is the optimal location also in cases (b) and (c), since the importance of having the resource in the different regions are strongly positively correlated. Compare Tables $2 \& 3$ If 
fire conditions are difficult in one of the countries in the southern region (IFPS), they are usually difficult also in the other countries in the south. Hence, there is often no reason to relocate resources from one country withing the region to another in the same region. The situation is the same within the northern region (FGLNS).

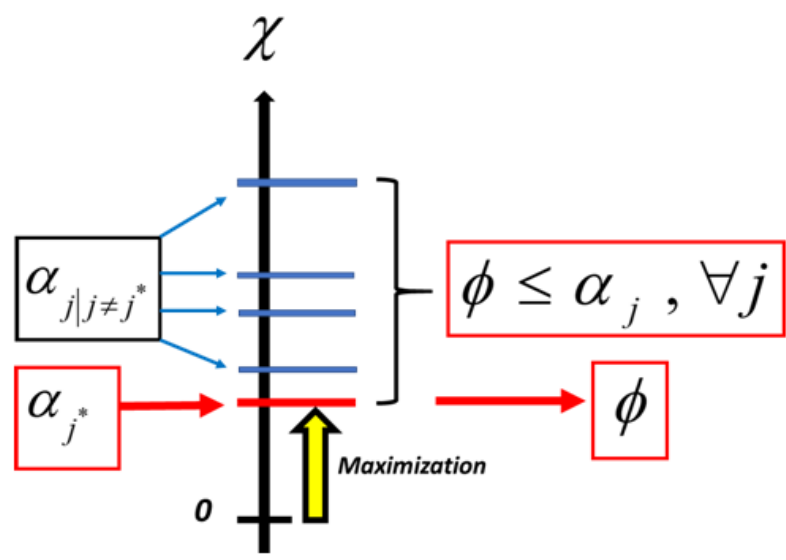

Figure 2 illustrates the linear programming approach to the optimization problem.

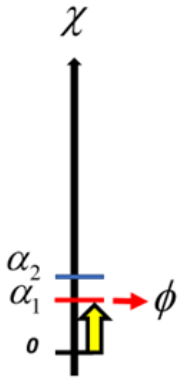

(a)

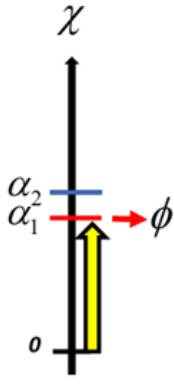

(b)

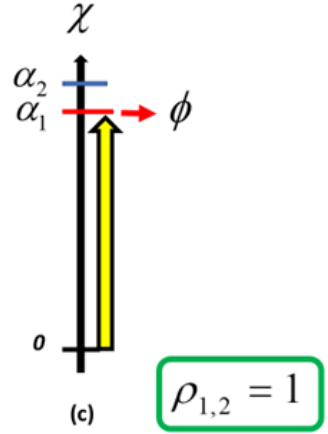

Figure 3 Optimization with two regions when the correlation is I.

Figure 4 illustrates the case where the correlation is zero. In two of the cases (a) and (b), we still prefer to use the mobile resources in region 1 . In the final case, however, it is better to send the resources to region 2.

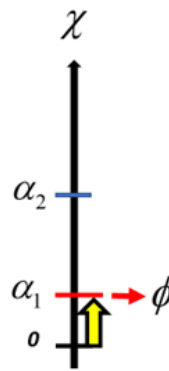

(a)

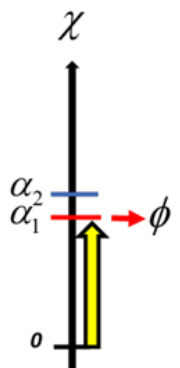

(b)

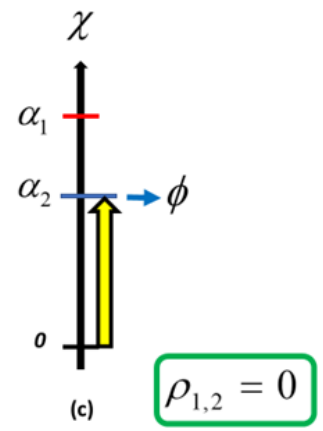

Figure 4 Optimization with two regions when the correlation is 0 .

Figure 5 shows what happens if we have strongly negative correlation between the fire problems in the different regions. When conditions are difficult in one region, they are often better in the other region. Hence, it is rather likely that the importance of using the resource in some region is high.

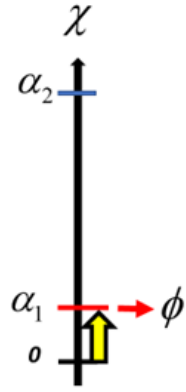

(a)

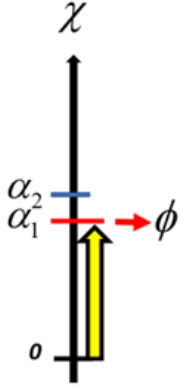

(b)

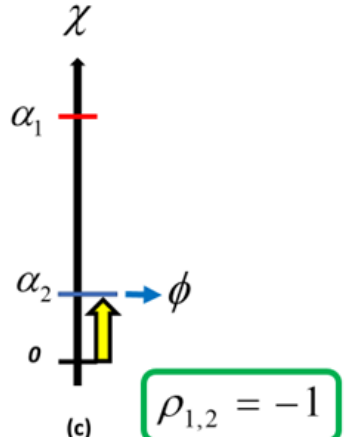

(c)
Figure 5 Optimization with two regions when the correlation is $-I$.

In equation (15), we state that the objective function is a nonstrictly increasing function of the correlation. This follows from the three Figures $3,4 \& 5$.

$$
\left(\Delta \rho_{1,2}>0\right) \Rightarrow(\Delta \phi \geq 0)
$$

\section{Special case:}

Is it possible that a relevant specification of the model implies that the expected value of the objective function is a strictly increasing function of the correlation of the fire problems in different countries or regions? In order to investigate this question, we start with a simplified version of the general problem. First, we limit the problem to two countries or regions. Then, we only need one correlation and do not need the indices.

$$
\rho=\rho_{1,2}
$$

We assume that the relationship is linear, the expected values are the same and that the standard deviations $\left(\sigma_{1}, \sigma_{2}\right)$ are equal. Compare Råde, Westergren, et al, 2004. ${ }^{16}$ The parameter $y$ connects equations (18) and (20).

$$
\begin{gathered}
E\left(\alpha_{2} \mid \alpha_{1}\right)=E\left(\alpha_{2}\right)+\rho \frac{\sigma_{2}}{\sigma_{1}}\left(\alpha_{1}-E\left(\alpha_{1}\right)\right) \\
E\left(\alpha_{2}\right)=E\left(\alpha_{1}\right)=y+\frac{1}{2}
\end{gathered}
$$

The probability density function is binary, as seen in (19). It is defined in equation (20).

$$
\begin{gathered}
f\left(\alpha_{1}\right) \in\{0,1\} \forall \alpha_{1} \\
f\left(\alpha_{1}\right)=\left\{\begin{array}{cc}
0 & \alpha_{1}<y \\
1 & y \leq \alpha_{1} \leq y+1 \\
0 & y+1<\alpha_{1}
\end{array}\right.
\end{gathered}
$$

We assume that the stochastic deviations (residuals) from the function (17) are small. Then, we can approximate $\alpha_{2}$ by the conditional expected value $E\left(\alpha_{2} \mid \alpha_{1}\right)$. This is seen in (21).

$$
\alpha_{2}=y+\frac{1}{2}+\rho\left(\alpha_{1}-y-\frac{1}{2}\right)
$$
(22).

The optimal objective function becomes the expression in equation

$$
\phi\left(\alpha_{1}, \alpha_{2}\left(\alpha_{1}\right)\right)=\min \left(\alpha_{1}, \alpha_{2}\left(\alpha_{1}\right)\right)
$$


In (23) and (24), we see how this depends on the parameters.

$$
\begin{gathered}
\phi= \begin{cases}\alpha_{1} & \alpha_{1}<y+\frac{1}{2} \\
\alpha_{2} & \alpha_{1} \geq y+\frac{1}{2}\end{cases} \\
\phi=\left\{\begin{array}{cc}
\alpha_{1} & \alpha_{1}<y+\frac{1}{2} \\
y+\frac{1}{2}+\rho\left(\alpha_{1}-y-\frac{1}{2}\right) & \alpha_{1} \geq y+\frac{1}{2}
\end{array}\right.
\end{gathered}
$$

Now, we investigate the expected optimal objective function value.

$$
E(\phi)=\int_{-\infty}^{\infty} \phi\left(\alpha_{1}, \alpha_{2}\left(\alpha_{1}\right)\right) f\left(\alpha_{1}\right) d \alpha_{1}
$$

Equations (20), (24) and (25) give (26).

$$
E(\phi)=\int_{y}^{y+\frac{1}{2}} \alpha_{1} d \alpha_{1}+\int_{y+\frac{1}{2}}^{y+1}\left(y+\frac{1}{2}+\rho\left(\alpha_{1}-y-\frac{1}{2}\right)\right) d \alpha_{1}
$$

In equations (27), (28) and (29), this is further developed.

$$
\begin{aligned}
& E(\phi)=\left[\frac{\left(\alpha_{1}\right)^{2}}{2}\right]_{y+0}^{y+\frac{1}{2}}+\left[\left(y+\frac{1}{2}-\rho y-\frac{1}{2} \rho\right) \alpha_{1}+\frac{1}{2} \rho\left(\alpha_{1}\right)^{2}\right]_{y+\frac{1}{2}}^{y+1} \\
& E(\phi)=\frac{1}{2}\left(\left(y+\frac{1}{2}\right)^{2}-y^{2}\right)+\left(y+\frac{1}{2}-\rho y-\frac{1}{2} \rho\right) \frac{1}{2}+\frac{1}{2} \rho\left((y+1)^{2}-\left(y+\frac{1}{2}\right)^{2}\right)
\end{aligned}
$$

\section{Results}

The expected optimal objective function value can be expressed as (29).

$$
E(\phi)=\frac{3}{8}+y+\frac{\rho}{8}
$$

We observe, in (30), that the expected optimal objective function value is a strictly increasing function of the correlation.

$$
\frac{d E(\phi)}{d \rho}=\frac{1}{8}>0
$$

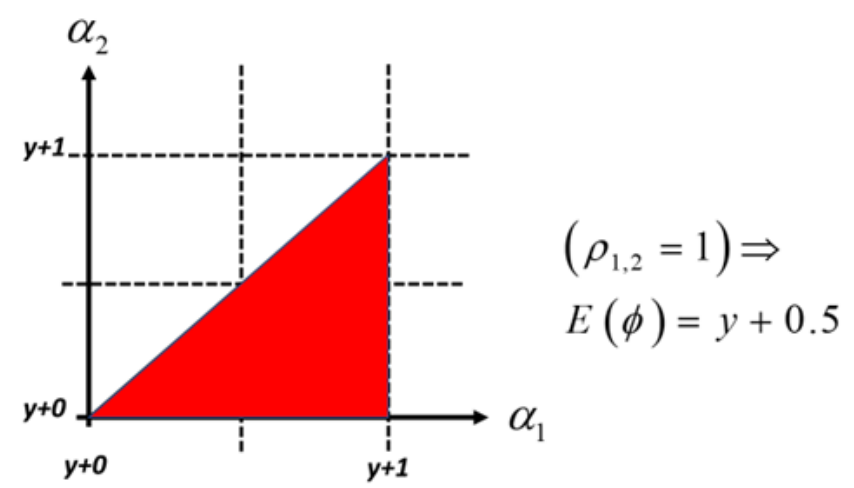

Figure $\mathbf{6}$ The expected objective function value, the red area plus $y$, with correlation I.

Equation (31) shows the expected optimal objective function values for three different correlation levels. Note that the Figures 6 ,
$7 \& 8$ present the same results via areas marked by red color. These areas should be interpreted as integrals.

$$
E(\phi)=\left\{\begin{array}{cl}
y+0.5 & \rho=1 \\
y+0.375 & \rho=0 \\
y+0.25 & \rho=-1
\end{array}\right.
$$

Finally, in Figure 9 we find a graph of the expected optimal objective value as a function of the correlation. ${ }^{14-16}$

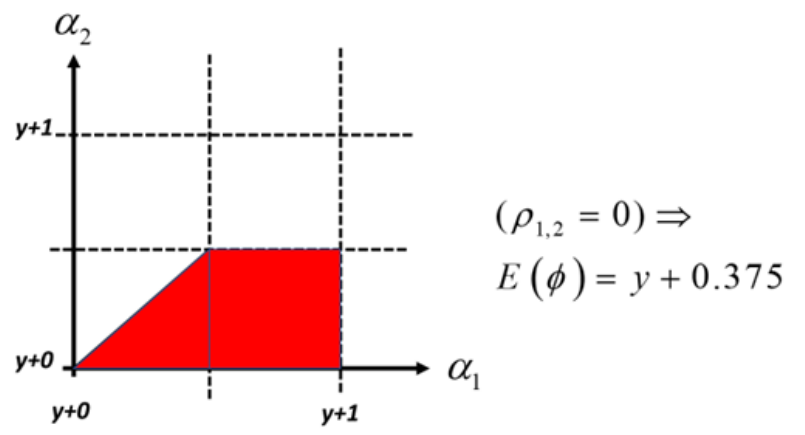

Figure 7 The expected objective function value, the red area plus $y$, with correlation 0 .

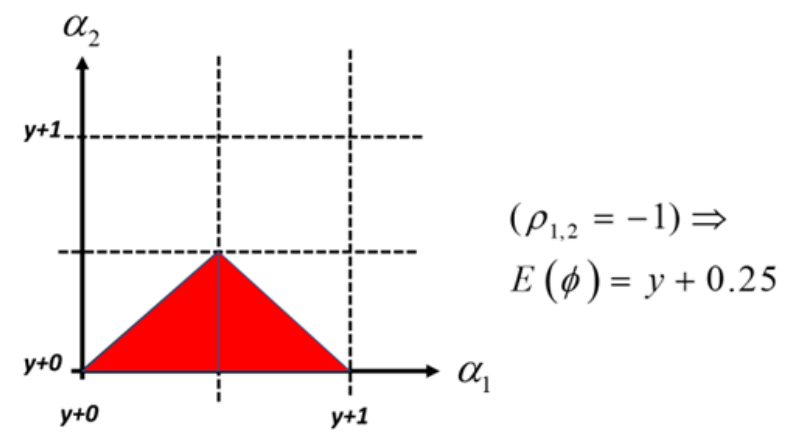

Figure 8 The expected objective function value, the red area plus $y$, with correlation - I.

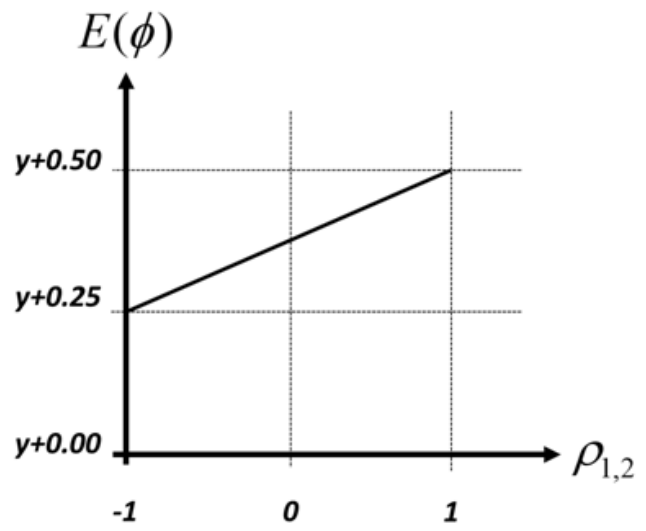

Figure 9 The expected objective function value as a function of the correlation.

\section{Discussion}

A model is just an approximation of some part of reality. The first model, defined in (1) - (4), is more general than the more specific analytical versions, found in equations (16)-(31). The ultimate goals 
of the models are two: a. To define and describe the system and 2. to derive some general conclusions concerning how the system should be controlled. The more general version (1) - (4) is useful when we have lots of empirical data available and want to create a precise numerical model. The more specific analytical models give us the option to understand general properties of the system and the optimal controls. The author suggests that future efforts to determine optimal strategies for international adaptive firefighting cooperation are based on explicit numerical versions of the defined optimization model according to equations (1), (2), (3) and (4). Without such analyses, expected costs will be higher, and fire problems worse, than necessary.

\section{Conclusions}

First, statistical properties of fires in different countries and regions in nine European countries were derived and described. It was found that two regions, IFPS and FGLNS, could be defined and that the fire correlations within the regions are negative and that the correlations between the regions are positive. This turned out to have important consequences for optimal international firefighting cooperation.

Allocation of internationally mobile firefighting units, such as water bombing airplanes, has been defined as a general multi period adaptive optimization problem. This was done via stochastic dynamic programming. Furthermore, a linear programming solution procedure was used to derive general conclusions. It was found that the objective function non-strictly decreases if the number of possible moves of the firefighting resources increases. This motivates expansion of international cooperation in firefighting operations. Furthermore, the objective function is a non-strictly increasing function of the correlation between the forest fire levels in different countries.

A specific version of the adaptive optimization problem was used to investigate the optimization problem is detail and to show how analytical solutions can be calculated. With this model, the expected optimal objective function value was determined as an explicit function of the correlation. It was proved that the expected optimal objective function value is a strictly increasing function of the correlation. Hence, since the lowest possible value of the expected objective function value is desired, the ideal situation is if the countries that share mobile firefighting resources have strongly negative fire correlation. Of course, the costs of moving equipment between nations is also an important factor.

Hence, as we understand from Table 3, adaptive firefighting cooperation between the regions IFPS and FGLNS have considerable advantages, thanks to the negative fire correlations. We must however not forget that the costs of moving equipment between some of these regions may be high. The costs of moving resources between the countries withing the regions IFPS and/or FGLNS are generally lower. Such moves are however usually less motivated since the degrees of fire problems have strongly positive correlations within the regions, as we see in Table 2.

\section{Funding}

None.

\section{Acknowledgments}

None.

\section{Conflicts of interest}

The authors declare that there is no conflict of interest.

\section{References}

1. FAO. Global Forest Resources Assessment 2020: Main report. Rome. 2020 .

2. Abatzoglou JT, Williams AP. Impact of anthropogenic climate change on wildfire across western US forests. Proceedings of the National Academy of Sciences of the United States of America. 2016;113(42):11770-11775.

3. Coogan SCP, Robinne FN, Jain P, et al. Scientists' warning on wildfire - a Canadian perspective. Canadian Journal of Forest Research. 2019; 49(9):1015-1023.

4. Williams AP, Abatzoglou JT, Gershunov A, et al. Observed Impacts of Anthropogenic Climate Change on Wildfire in California. Earth's Future. 2019.

5. Lohmander P. Dynamics and control of the CO2 level via a differential equation and alternative global emission strategies. International Robotics \& Automation Journal, 2020;6(1):7-15.

6. Lohmander P. Fundamental principles of optimal utilization of forests with consideration of global warming. Central Asian Journal of Environmental Science and Technology Innovation. 2020;1(3):134-142.

7. Lohmander P. Optimization of continuous cover forestry expansion under the influence of global warming. International Robotics \& Automation Journal. 2020;6(3):127-132.

8. San-Miguel-Ayanz J, Durrant T, Boca R, et al. Forest Fires in Europe, Middle East and North Africa 2018. European commission. 2019.

9. Cave D. The World Burns All Year. Are There Enough Planes to Douse the Flames?. The New York Times. 2019.

10. The Maritime Executive. Canada and Australia Could Transport Water Bombers by Sea. The Maritime Executive. 2020.

11. Fire \& Rescue World News. Italian water bombers on standby to assist Scandinavia's forest fire efforts. International Association of Fire and Rescue Services. 2018.

12. Mason R. Sweden's wildfire defense expands with air tractor scooper water bombers. The Aerial Firefighting Magazine. 2020.

13. Winston WL. Operations Research - Applications and algorithms (with CD-ROM and InfoTrac). Thomson, Brooks/Cole, $4^{\text {th }}$ edition. 2004.

14. Lohmander P. Two Approaches to Optimal Adaptive Control under Large Dimensionality. International Robotics and Automation Journal. 2017;3(4):328-330.

15. Lohmander P. Applications and Mathematical Modeling in Operations Research. In: Cao BY, editor. Fuzzy Information and Engineering and Decision. IWDS 2016. 2018.

16. Råde L, Westergren B. Mathematics Handbook for Science and Engineering. Springer. 1999. 